\title{
The predictive value of transabdominal ultrasonography in the diagnosis of biliary tract complications after orthotopic liver transplantation
}

\author{
S H Hussaini, M B Sheridan, M Davies
}

\begin{abstract}
Background-In transplant recipients with choledococholedocostomy (CDCD), endoscopic retrograde cholangiopancreatography (ERCP) remains the gold standard for the diagnosis of biliary leak or strictures. Transabdominal ultrasonography (TAUS) has been used to screen patients with suspected biliary tract complications, prior to ERCP, although the clinical effectiveness remains unclear.

Aims-To assess the predictive value of TAUS in the diagnosis of biliary tract complications after liver transplantation. Methods-144 consecutive ERCP and corresponding ultrasonogram reports performed over a 67 month period in 79 patients after liver transplantation were analysed retrospectively.

Results-77 ERCP patients had both a TAUS and a successful ERCP. Biliary tract abnormalities were found at TAUS in $49(64 \%)$ of the 77 patients. TAUS had an overall sensitivity of $77 \%$, and specificity of $67 \%$, with positive and negative predictive values of $26 \%$ and $95 \%$ respectively, when adjusted for the prevalence rate of biliary complications after liver transplantation of $12.8 \%$ in our population. The use of bile duct calibre as sole criterion for an abnormal scan improved the specificity $(76 \%)$ and with a corresponding reduction in sensitivity $(66 \%)$. The risk of false negative TAUS was similar in both the early and late post-transplant periods. Conclusions-A normal TAUS after liver transplantation with CDCD makes the presence of biliary complications unlikely. (Gut 1999;45:900-903)
\end{abstract}

Keywords: endoscopic retrograde

cholangiopancreatography; transabdominal ultrasonography; orthotopic liver transplantation; predictive value; sensitivity; specificity

Biliary tract complications after orthotopic liver transplantation are a cause of considerable morbidity and mortality. ${ }^{12}$ The advent of modified techniques of biliary reconstruction such as choledococholedocostomy (CDCD), ${ }^{3}$ increasingly without the use of a $\mathrm{T}$ tube stent, ${ }^{45}$ has reduced the incidence of biliary complications after liver transplantation. ${ }^{67}$

Biliary complications after transplantation have clinical and laboratory features similar to those of acute or chronic rejection, sepsis, post-transplant hepatitis, or hepatic artery occlusion. $^{7}$ In transplant recipients with CDCD, endoscopic retrograde cholangiopancreatography (ERCP) remains the gold standard for the diagnosis of biliary leak or strictures. However, ERCP is an invasive investigation with a small but significant morbidity and mortality. Thus transabdominal ultrasonography (TAUS) has been used to screen patients with possible biliary tract complications. ${ }^{8}$ Unfortunately, TAUS is associated with false negative results, ${ }^{9-11}$ although these data refer to earlier transplant series when CDCD was uncommon. Thus the aim of the current study was to assess the predictive value of TAUS in the diagnosis of biliary tract complications prior to ERCP.

\section{Methods}

DATA ACQUISITION

Patients were identified retrospectively from the Radiology Department database, as those who underwent ERCP between October 1991 and April 1997 following liver transplantation at St James's University Hospital. In total, 144 consecutive ERCPs performed in 79 patients were identified, 43 of whom had a single procedure. A total of 101 ERCPs was performed in the remaining 36 patients. The mean number of ERCPs per patient was two (range 1-5, median 1). In 10 patients, repeat ERCP was performed for a clinical indication which was distinct from the index episode, such as an episode of possible biliary obstruction in a second liver graft. In the remaining 26 individuals who underwent multiple ERCPs, the indication for repeat ERCP was for stent placement, removal, or replacement, and for failed initial cannulation of the common bile duct; these repeat ERCPs were excluded from the studies of the predictive value of TAUS. Thus a total of 89 ERCPs from 79 patients was suitable for data analysis. However, 12 of these 89 ERCPs were excluded from data analysis for the following reasons: no ultrasound before ERCP $(n=8)$; no cholangiogram obtained at ERCP $(n=3)$; or no cholangiogram obtained at ERCP or TAUS performed pre-ERCP $(n=1)$. Therefore, in all, there were 77 evaluable cases of

Abbreviations used in this paper: $\mathrm{CDCD}$, choledococholedocostomy; ERCP, endoscopic retrograde cholangiopancreatography; PTC, percutaneous transhepatic cholangiography; TAUS, transabdominal ultrasonography. 


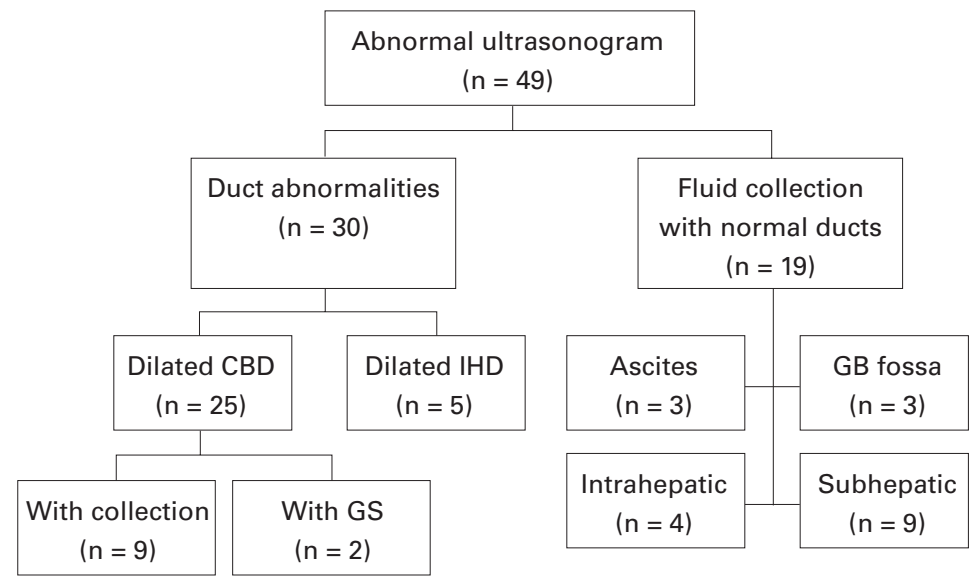

Figure 1 Details of ultrasonographic abnormalities found in the patients studied before ERCP with the numbers of patients in parentheses. CBD, common bile duct; IHD, intrahepatic duct dilatation; GB, gall bladder; GS, gallstones.

TAUS and ERCP cholangiogram results available to determine the predictive value of TAUS with regard to biliary complications.

The corresponding ultrasonogram reports were retrieved for further unblinded review, with the ERCP cholangiogram films, by a consultant radiologist (MBS) with an interest in biliary radiology. Measurements of common bile duct calibre were made on every patient, according to a set protocol for liver transplant recipients. All ultrasound scans were not performed by one person, but by sonographers trained in the department or by radiologists. The case notes and liver function tests were then reviewed to determine the clinical indications for ERCP.

PATIENTS

A total of 42 male (mean age 46 (SEM 7.1) years) and 37 female (mean age 49 (SEM 8.0) years), transplant recipients was studied. Between October 1991 and April 1997, 365 transplant procedures were performed in 308 patients, of whom 70 underwent subsequent ERCP. Thus nine of the patients studied were transplanted prior to the setting up of the radiology database, but underwent ERCP during the study period. These nine patients transplanted prior to April 1991 were included in the study, as the primary aim of the study was not to define the incidence of biliary complications, but to determine the predictive value of TAUS.

BILIARY RECONSTRUCTION

A total of 75 patients underwent an "end to end" biliary anastomosis, with $\mathrm{T}$ tube placement in 19 cases. Three patients had a gall bladder conduit and one patient underwent primary Roux loop biliary reconstruction, all transplanted before April 1991.

RADIOLOGICAL CRITERIA FOR BILIARY

COMPLICATIONS

The TAUS criteria taken to be suggestive of biliary complications and regarded as a positive ultrasonogram were: (1) dilated intrahepatic ducts; (2) common bile duct diameter greater than $7 \mathrm{~mm}$; (3) intrahepatic collections; and/or (4) hilar and perihepatic collections.
ERCP was taken as the gold standard for a "true positive" with a cholangiogram which had the following radiological findings: (1) biliary stricture (anastomotic or nonanastomotic); (2) leak of contrast; (3) sludge and/or stones; and (4) biliary dilatation (greater than the calibre of the duodenoscope) with no obstructive lesion. The median time between biliary TAUS and ERCP was five days (range $1-71$ days).

STATISTICS

The usefulness of TAUS in the detection of biliary complications after liver transplantation was initially assessed by calculating the sensitivity (percentage of true positive scans correctly identified by TAUS) and specificity (percentage true negative scans correctly identified by TAUS) using the cholangiogram obtained at ERCP as the gold standard. The predictive value of TAUS was calculated as the percentage of patients with an abnormal scan who had an abnormal cholangiogram (positive predictive value) and percentage of patients with a normal scan who had a normal cholangiogram (negative predictive value). The predictive value was then adjusted for the prevalence rate of biliary complications. ${ }^{12}$ The TAUS results were analysed using all positive criteria and using biliary dilatation as the sole positive criterion. The predictive value of TAUS was also analysed in the early (ERCPs performed within 100 days) and late (ERCPs performed after 100 days) periods after transplantation.

The difference in predictive value in the early and late transplant periods was compared using Fisher's exact test. The level of significance was set at $\mathrm{p}<0.05$. Results were expressed as mean values (SEM) or medians as appropriate. Data were analysed using StatCalc, version 5 and Excel, version 5.0.

\section{Results}

CLINICAL INDICATIONS FOR ERCP

A total of 49 abnormal TAUS was reported prior to ERCP, the details of which are shown in fig 1. Intrahepatic or extrahepatic biliary dilatation was observed in $61 \%$ of patients with an abnormal TAUS, although the remaining patients had suspicious intra-abdominal fluid collections with normal duct calibre.

A normal TAUS was reported before ERCP in 28 patients. The major indications for ERCP in these patients were as follows: cholestatic liver function tests with a serum bilirubin greater than $100 \mu \mathrm{mol} / 1$ and/or alkaline phosphatase greater than $500 \mathrm{IU} / 1(\mathrm{n}=24)$; hepatic artery occlusion shown by angiogram $(n=1)$;

Table 1 Comparison of transabdominal ultrasonography (TAUS) and endoscopic retrograde cholangiopancreatography (ERCP) findings

\begin{tabular}{lll}
\hline & \multicolumn{2}{l}{ ERCP } \\
\cline { 2 - 3 } & Abnormal & Normal \\
\hline TAUS & & \\
Abnormal & $41(84 \%)$ & $8(16 \%)$ \\
Normal & $12(43 \%)$ & $16(57 \%)$ \\
\hline
\end{tabular}


Table 2 Predictive value of transabdominal ultrasonography

\begin{tabular}{|c|c|c|c|c|c|c|}
\hline & \multirow{2}{*}{$\begin{array}{l}\text { Sensitivity } \\
(\%)\end{array}$} & \multirow{2}{*}{$\begin{array}{l}\text { Specificity } \\
(\%)\end{array}$} & \multicolumn{2}{|c|}{ Crude predictive value (\%) } & \multicolumn{2}{|c|}{ Adjusted predictive value (\%) } \\
\hline & & & Positive & Negative & Positive & Negative \\
\hline Overall & 77 & 67 & 84 & 57 & 26 & 95 \\
\hline Dilated ducts & 66 & 76 & 83 & 55 & 29 & 94 \\
\hline ERCP $<100$ days & 81 & 64 & 86 & 54 & 25 & 96 \\
\hline $\mathrm{ERCP}>100$ days & 73 & 62 & 76 & 57 & 22 & 94 \\
\hline
\end{tabular}

ERCP, endoscopic retrograde cholangiopancreatography.

an abnormal $\mathrm{T}$ tube cholangiogram $(\mathrm{n}=2)$; and cholangitis alone $(n=1)$.

ERCP FINDINGS AFTER TRANSPLANTATION

Biliary tract abnormalities were found at ERCP in 53 of the 77 patients analysed. The cholangiogram performed at ERCP was normal in 24 patients $(31 \%)$. A total of 41 patients had a biliary stricture, 36 of which were anastomotic, two anastomotic with associated biliary leaks, two with combined anastomotic and non-anastomotic, and one nonanastomotic stricture alone. Common duct dilatation was seen in five patients, one of whom had duct dilatation with small common bile duct stones. A further three patients had biliary sludge, casts, or stones in a non-dilated biliary system. Finally, four patients had biliary leaks.

PREDICTIVE VALUE OF TRANSABDOMINAL ULTRASONOGRAPHY

Table 1 gives the number of abnormal and normal ultrasonograms performed after transplantation, using ERCP as the gold standard. Table 2 gives the data for sensitivity, specificity, and crude and adjusted predictive values for transabdominal ultrasonograms in the detection of biliary abnormalities after transplantation. In those patients transplanted between October 1991 and April 1997, the prevalence of biliary complications in patients with CDCD was $12.8 \%$. This prevalence rate was used to calculate the adjusted predictive value of TAUS for the current cohort of patients.

TAUS was excellent at predicting an abnormal cholangiogram. Only $16 \%$ of the patients in this study, whose TAUS was abnormal, had a normal cholangiogram. However, in the transplant population the prevalence of biliary complications was $12.3 \%$; thus the adjusted positive predictive value was only $26 \%$, and the false positive predictive value was as high as $74 \%$. Therefore, $74 \%$ of transplant recipients with an abnormal TAUS are likely to have a normal cholangiogram at ERCP. This is an inevitable result of the low prevalence rate of biliary complications and not a reflection of the sensitivity or specificity of ultrasound.

In contrast, TAUS incorrectly predicted a normal cholangiogram in $43 \%$ of patients, an apparent high false negative rate. However, this rate fell dramatically, when adjusted for the low prevalence rate of biliary complications to only $5 \%$. Thus only $5 \%$ of the transplant population with a normal TAUS are likely to have abnormal cholangiograms at ERCP. The use of bile duct calibre as sole criterion for an abnormal scan improved the specificity, with a corresponding reduction in sensitivity. The adjusted negative predictive value of TAUS was similar in patients with and without dilated ducts, and in those patients whose ERCPs were performed within 100 and 100 days after liver transplantation.

A total of 12 false negative scans occurred: an equal number in the early (within 100 days) and late (after 100 days) periods after transplantation. Three were due to missed small stones, sludge, and casts in the common bile duct. One patient in the early and one in the late post-transplant period had nonobstructive common duct dilatation at cholangiography, with a normal scan before ERCP. Four anastomotic strictures were missed by TAUS within 100 days compared with three (one non-anastomotic) after 100 days.

\section{Discussion}

End to end CDCD is the preferred biliary anastomosis in $75 \%$ of adult liver transplant operations. ${ }^{13}$ CDCD without $\mathrm{T}$ tube drainage $^{45}$ is often performed, and therefore TAUS, followed by ERCP, is currently used to evaluate and treat biliary complications arising after liver transplantation. ${ }^{13}$ The current study is unique in that the clinical effectiveness of TAUS has been evaluated in a large number of post-transplant patients from a single centre who underwent CDCD in the majority of cases. We have shown that TAUS after liver transplantation with CDCD has good predictive value for biliary complications.

The presence of fever, ascites, cholestatic liver function tests, and jaundice are suggestive of biliary complications, although the differential diagnosis includes acute and chronic rejection, hepatic artery occlusion, and posttransplantation hepatitis. We used Doppler ultrasonography to establish the integrity of the hepatic vascular supply and biliary tree. Nonetheless, patients underwent ERCP, despite the finding of a normal transabdominal ultrasonogram, if there was clinical suspicion of biliary complications. The rationale for this diagnostic approach was the poor sensitivity of TAUS after liver transplantation in the detection of biliary complications documented in earlier reports. ${ }^{11}{ }^{14}$ Therefore, although the current study was retrospective and thus subject to selection bias, we believe that the sensitivity and specificity of TAUS as a screening investigation has been validly assessed, as the diagnostic algorithm used during this study did not assume that TAUS was a reliable indicator of biliary complications.

The sensitivity of TAUS in the detection of biliary complications in the present series was considerably greater than in two early studies which reported sensitivity rates between 38\% 
and $45 \% .{ }^{11}{ }^{14}$ We speculate that the use of $\mathrm{T}$ tube drainage in these earlier studies may have reduced the diagnostic effectiveness of TAUS. Moreover, we deliberately examined the use of ultrasound in patients prior to ERCP, rather than percutaneous transhepatic cholangiography (PTC). Patients undergoing PTC will usually have a Roux-en Y biliary reconstruction which may also limit the predictive value of ultrasound, as there is no extrahepatic biliary system to examine.

The negative predictive value of TAUS in the detection of biliary complications for a given individual was only $57 \%$, which although better than earlier reports, ${ }^{14}$ is a cause for concern if TAUS is to be used as a screening technique. We found that the prevalence of biliary complications after liver transplantation was $12.8 \%$ of patients with a CDCD. Our results and those from other groups ${ }^{31315}$ highlight the fact that the incidence of biliary complications has fallen in recent years. The low overall prevalence of biliary complications has practical implications for TAUS when used as a screening investigation in clinical practice. The predictive value of any investigation is not only determined by the sensitivity and specificity of the investigation, but also the prevalence of the screened abnormality. ${ }^{12}$ Thus, as biliary complications after liver transplantation become more infrequent, one can be more certain that a negative transabdominal ultrasonogram truly indicates that there is no biliary pathology. Therefore, although the negative predictive value of TAUS for an individual was only $57 \%$, because the prevalence of biliary complications is relatively low, a negative scan more or less excludes biliary complications.

The risk of false negative transabdominal ultrasonograms seems to be similar in both the early and late post-transplant periods. The false negative scans arose for a number of reasons. Firstly, TAUS failed to detect small stones, sludge, and casts, as has been reported previously. ${ }^{10}$ Secondly, two patients had biliary dilatation alone, with no obstruction at cholangiography, although the significance of this finding is unclear. Finally, the delay in the time between ultrasound and ERCP may have accounted for the false negative TAUS in three of the seven patients with biliary strictures in whom the cholangiogram was obtained more than six days after TAUS.

In conclusion, we found that TAUS is a sensitive and specific method for the detection of biliary complications after liver transplantation. Indeed, when the incidence of biliary complications is relatively low, a normal scan after liver transplantation with an "end to end" biliary anastomosis makes the presence of biliary complications very unlikely.

This work was presented at the 6th UEGW meeting in Birmingham, 1997 and published in abstract form (Gut 1997;41:A78)

1 Lerut J, Gordon RD, Iwatsuki S, et al. Biliary tract complications in human liver transplantation. Transplantation 1987;43:47-51.

2 Van Thiel DH, Fagiuoli S, Wright HI, et al. Biliary complications of liver transplantation. Gastrointest Endosc 1993;39: 455-60.

3 Gholson CF, Zibari G, McDonald JC. Endoscopic diagnosis and management of biliary complications following orthotopic liver transplantation. Dig Dis Sci 1996;41:104553

4 Rouch DA, Emond IC, Thislethwaite JR, et al. Choledochocholedochostomy without a $\mathrm{T}$ tube or internal stent in transplantation of the liver. Surg Gynecol Obstet 1990;170: 239-44

5 Rolles K, Dawson K, Novell R, et al. Biliary anastomosis following liver transplantation does not benefit from $\mathrm{T}$ tube splintage. Transplantation 1994;57:402-4.

6 Evans RA, Raby ND, O'Grady JG, et al. Biliary complications following orthotopic liver transplantation. Clin Radiol 1990;41:190-4.

7 Gomes AS. Diagnosis and radiological treatment of biliary complications of liver transplantation. Semin Interv Radiol 1992;9:283-9.

8 Morton MJ, James EM, Wiessner RH, et al. Applications of duplex ultrasonography in the liver transplant patient. Mayo Clin Proc 1990;65:360-7.

9 Zemel G, Zajko AB, Skolnick ML, et al. The role of sonography and transhepatic cholangiography in the diagnosis of biliary complications after liver transplantation. $A f R A m$ $\mathcal{F}$ Roentgenol 1988;151:943-6.

10 O'Connor HI, Vickers CR, Buckels JAC, et al. Role of endoscopic retrograde cholangiopancreatography after orthotopic liver transplantation. Gut 1991;32:419-23.

11 Zajko AB, Zemel G, Skolnick ML, et al. Percutaneous transhepatic cholangiography rather than ultrasound: a screening test for postoperative biliary complications in liver transplant patients. Transplant Proc 1988;22(suppl):67881.

12 Altman DG, Bland JM. Diagnostic tests 2: predictive values. BM7 1994;309:102.

13 Sossenheimer M, Slivka A, Carr-Locke D. Management of extrahepatic biliary disease after orthotopic liver transplantation: review of the literature and results of a transplantation: review of the literature and

14 Kok T, Van der Sluis A, Klein JP, et al. Ultrasound and cholangiography for the diagnosis of biliary complications after orthotopic liver transplantation: a comparative study. f Clin Ultrasonography 1996;24:103-15.

15 Grief F, Bronsther OL, Van Thiel DH, et al. The incidence, timing and management of biliary complications after orthotopic liver transplantation. Ann Surg 1994;219:40-5. 